\title{
The surgical perspective in neoadjuvant immunotherapy for resectable non-small cell lung cancer
}

\author{
Long Jiang ${ }^{1}$ (1) · Jia Huang ${ }^{1}$. Shanshan Jiang ${ }^{2} \cdot$ Wenwen Rong $^{3} \cdot$ Yaofeng Shen ${ }^{4} \cdot$ Chongwu Li $^{1} \cdot$ Yu Tian ${ }^{1}$. \\ Junwei Ning ${ }^{1}$ - Xiaoke Chen ${ }^{1}$. Yunhai Yang ${ }^{1}$. Zhengping Ding ${ }^{1} \cdot$ Ziming $\mathrm{Li}^{1}$ · Qingquan Luo ${ }^{1}$
}

Received: 20 June 2020 / Accepted: 2 January 2021 / Published online: 29 January 2021

(c) The Author(s), under exclusive licence to Springer-Verlag GmbH, DE part of Springer Nature 2021

\begin{abstract}
Background The recent novel conception of neoadjuvant immunotherapy has generated interest among surgeons worldwide, especially the lack of experience involving surgical treatment for the neoadjuvant immunotherapy population.

Methods Patients with non-small cell lung cancer (NSCLC), who underwent neoadjuvant immunotherapy or chemo-immunotherapy, were retrospectively collected between September 2018 and April 2020. Demographic data, pathological and clinical features, therapeutic regimens and outcome data of all individuals were collected by retrospective chart review. Operative details, information of neoadjuvant therapy, were also abstracted.

Results In total, 31 patients were included in the final analysis. The patients' median age was 61 years. In total, 29 of the patients were males, while 2 were females. Patients received a median of 3 doses before resection. The median duration from final treatment to surgery was 34 days. After neoadjuvant treatment, post-treatment computed tomography scan showed that 24 patients had partial response. In total, 12 of 31 patients had a major pathological response, 15 pathological downstaging. Three patients had no residual viable tumor. A positive surgical margin was identified in 7 cases. One or more postoperative complications occurred in 18 of all 31 patients. In total, 26 patients underwent next-generation sequencing before surgery in total. Among them, 2 patients were detected STK11 mutations, none of whom had a major pathological response by final pathological examination.
\end{abstract}

Conclusions Pulmonary resection after neoadjuvant immunotherapy or chemo-immunotherapy for resectable NSCLC appears to be safe with low operative mortality and morbidity rate in the current population.

Keywords Neoadjuvant treatment $\cdot$ Immunotherapy $\cdot$ Non-small cell lung cancer

Long Jiang, Jia Huang, Shanshan Jiang, Wenwen Rong and Yaofeng Shen have contributed equally to this work.

Qingquan Luo

luoqingquan@hotmail.com

1 Shanghai Lung Cancer Center, Shanghai Chest Hospital, Shanghai Jiaotong University, Shanghai 200030, China

2 State Key Laboratory for Oncogenes and Related Genes, Key Laboratory of Gastroenterology and Hepatology, Ministry of Health, Division of Gastroenterology and Hepatology, Renji Hospital, School of Medicine, Shanghai Jiao Tong University, Shanghai Cancer Institute, Shanghai Institute of Digestive Disease, 145 Middle Shandong Road, Shanghai 200001, China

3 Statistical Center, Shanghai Chest Hospital, Shanghai Jiaotong University, Shanghai 200030, China

4 Department of Anesthesiology, Shanghai Chest Hospital, Shanghai Jiaotong University, Shanghai 200030, China

\section{Introduction}

Globally, lung cancer is one of the most common malignancies, with its high incidence, mortality and low 5-year survival rate [1]. Non-small cell lung cancer (NSCLC) is responsible for more than $85 \%$ of all cases of lung cancer, and approximately one-third of NSCLC cases are diagnosed at a locally advanced stage [2]. For patients with early stage (stage I and II) and appropriately selected locally advanced disease (stage IIIA and IIIB), the standard approach is multidisciplinary treatment including complete surgical resection, chemotherapy, radiotherapy, targeted therapy and so on [3].

Traditionally, platinum-based chemotherapy is mainly used in advanced or metastatic NSCLC [4]. More recently, PD-(L)1 immune checkpoint inhibitors have changed the treatment paradigm of patients with advanced or metastatic NSCLC [5, 6]. The development of anticancer 
immunotherapy targeting PD-1/PD-L1 and CTLA-4 immune checkpoints has achieved encouraging therapeutic effects in many cancers, including melanoma, NSCLC, renal cell cancer and Hodgkin disease [7]. With evidence that targeting the host immune response to lung cancer, and development of immune checkpoint blockage agents, immunotherapy has become the first-line therapy for the majority of patients with metastatic NSCLC [8].

Neoadjuvant therapy in the treatment of NSCLC is well established as part of the guidelines [9]. Recent published pilot data on preoperative use of checkpoint inhibition in patients with NSCLC suggested the safety and feasibility of inductive immunotherapy with surgically resectable cases [10-17]. The novel conception of neoadjuvant immunotherapy has generated interest among surgeons worldwide [18]. However, the lack of experience involving surgical treatment for the neoadjuvant immunotherapy population, caused substantial concerns of surgical perspective regarding the inflammatory action of these agents, such as pneumonitis and endocrinopathies, and subsequent technical challenges [19]. To address this, the current study was conducted to report the analysis of surgical perspective outcome data after neoadjuvant immunotherapy followed by surgery for resectable NSCLC.

\section{Patients and methods}

The current retrospective study was conducted at Shanghai Chest Hospital, an ultra-high-volume tertiary thoracic surgery center in Shanghai, China. The institutional review board approved the retrospective analysis of anonymous patient data. The data were retrospectively collected on patients admitted to Shanghai Chest Hospital between September 2018 and April 2020. Patients with histologic diagnosis of NSCLC underwent neoadjuvant immunotherapy or chemo-immunotherapy and with available clinicopathological characteristics were included. Exclusion criteria included patients recruited in blind clinical trials, unavailable clinicopathological characteristics.

The treatment regimens, including initial and subsequent regimens, indication for surgery were always discussed and determined by a multidisciplinary team. Demographic data, pathological and clinical features, therapeutic regimens and outcome data of all individuals were collected by retrospective chart review.

For all patients, preoperative assessment approaches should contain contrast-enhanced computed tomography (CT) scan or positron emission tomography (PET)/CT scan, brain imaging with $\mathrm{CT}$ or magnetic resonance imaging, pretreatment tumor biopsy, invasive mediastinal nodal staging with endobronchial ultrasound or mediastinoscopy. Operative details, including operative approach, extent of resection, operative time, hospital length of stay, postoperative morbidity, rationale for conversion of minimally invasive surgical procedures to thoracotomy if necessary and other details related to the operative experience, were recorded in detail. Information of neoadjuvant therapy, such as agents, courses, duration of final neoadjuvant treatment to surgery, was also abstracted.

Patients were staged according to AJCC Lung Cancer Staging, 8th edition (2017) [20]. Operative time was obtained by review of the anesthesia and operating room nurse record, which was defined as the time from skin incision to closure. Surgical complications were evaluated and recorded according to the criteria defined by the Society of Thoracic Surgeons and the European Society of Thoracic Surgeons general thoracic surgery databases [21]. Briefly, complete pathological response was defined as $0 \%$ viable tumor cells in residual tumor, while major pathological response as $10 \%$ remaining [22].

\section{Statistical analysis}

Data were expressed as median and range unless otherwise indicated, and $P<0.05$ was considered significant. Statistical calculations were conducted with SPSS software (IBM SPSS Statistics for Windows, version 22.0., IBM Corp., Armonk, NY, USA).

\section{Result}

Of the 14,901 patients underwent lung resection in our institute during the study period, 31 patients received neoadjuvant immunotherapy or chemo-immunotherapy for at least 2 courses were included in the study. Majority of these 14,901 patients were treated with surgery only, instead of adjuvant or neoadjuvant treatment, due to their early-stage diseases. The demographic and clinical characteristics of the overall cohort are listed in Table 1. The patients' median age at the time of the surgery was 61 years (range, 38-77 years). In total, 29 of the patients were males $(93.5 \%)$, while 2 were females (6.5\%). Squamous cell carcinoma $(n=22,71.0 \%)$ was the most common histologic subtype and adenocarcinoma $(n=9,29.0 \%)$ second most common. In total, the majority of patients had stage IIIA disease $(16,51.6 \%)$, and 10 patients $(32.3 \%)$ with stage IIIB disease, $4(12.9 \%)$ with stage IIB disease, $1(3.2 \%)$ with stage IIA disease at the stage of treatment naive. Moreover, pathological responses were observed regardless of tumor PD-L1 expression, and PD-L1 expression did not appear to be predictive of a treatment benefit (Table 1, Fig. 1).

None of the patients received preoperative radiotherapy. The most commonly used neoadjuvant regimens 
Table 1 Demographic and clinical characteristics of the overall cohort

\begin{tabular}{|c|c|c|c|}
\hline Characteristic & $<=10 \%$ viable tumor $(\mathrm{n}=12)$ & $>10 \%$ viable tumor $(n=19)$ & $p$ value \\
\hline Median age (range), ys & $60(48-77)$ & $61(38-66)$ & 0.408 \\
\hline Sex & & & 0.510 \\
\hline Female & 0 & 2 & \\
\hline Male & 12 & 17 & \\
\hline Median BMI (range) & $23.81(21.05-27.77)$ & $24.49(17.72-28.39)$ & 0.805 \\
\hline Clinical stage & & & 0.455 \\
\hline IIA & 0 & 1 & \\
\hline T2bN0 & 0 & 1 & \\
\hline IIB & 3 & 1 & \\
\hline T2aN1 & 1 & 1 & \\
\hline T2bN1 & 1 & 0 & \\
\hline T3N0 & 1 & 0 & \\
\hline IIIA & 6 & 10 & \\
\hline T2aN2 & 3 & 5 & \\
\hline $\mathrm{T} 2 \mathrm{bN} 2$ & 0 & 1 & \\
\hline T3N1 & 2 & 1 & \\
\hline T4N0 & 0 & 2 & \\
\hline T4N1 & 1 & 1 & \\
\hline IIIB & 3 & 7 & \\
\hline $\mathrm{T} 3 \mathrm{~N} 2$ & 3 & 3 & \\
\hline $\mathrm{T} 4 \mathrm{~N} 2$ & 0 & 4 & \\
\hline Histologic subtype & & & 0.101 \\
\hline Squamous cell carcinoma & 11 & 11 & \\
\hline Adenocarcinoma & 1 & 8 & \\
\hline Smoking history & & & 0.523 \\
\hline Never & 8 & 16 & \\
\hline Former & 3 & 1 & \\
\hline Current & 1 & 2 & \\
\hline PD-L1 expression & & & 0.352 \\
\hline$<1 \%$ & 5 & 5 & \\
\hline $1-50 \%$ & 1 & 4 & \\
\hline$>50 \%$ & 2 & 5 & \\
\hline NA & 4 & 5 & \\
\hline
\end{tabular}

$B M I$ body mass index, NA Unknown
Fig. 1 Detailed information involving radiographic response, pathological response, pretreatment PD-L1 expression and NGS result of all 31 patients. $N G S$ next-generation sequencing; $P R$ partial response;

were chemo-immunotherapy $(n=27,87.1 \%)$, followed by immunotherapy alone $(n=4,12.9 \%)$. The most commonly prescribed checkpoint inhibitor was pembrolizumab (16, $51.6 \%)$, the others as nivolumab $(15,48.4 \%)$. The frequencies of the most commonly used drugs and drug classes are shown in Table 2. Patients received a median of 3 doses before resection (range, 2-4 doses). The median duration from final treatment to surgery was 34 days (range,
$S D$ stable disease; $P D$ progressive disease; $p C R$ pathological complete remission; $N A$ not applicable

4-93 days). After neoadjuvant treatment, post-treatment CT scan showed that 24 patients (77.4\%) had partial response, five patients $(16.1 \%)$ stable disease and two $(6.5 \%)$ radiographic progression of disease (Table 2 and Fig. 1).

Details of surgical intervention and tumor location for the patients are listed in Table 3. The most common procedure was lobectomy $(18,58.1 \%)$, followed by sleeve lobectomy $(7,22.6 \%)$, bilobectomy $(4,12.9 \%)$ and pneumonectomy 
Table 2 Neoadjuvant characteristics of the overall cohort

\begin{tabular}{llll}
\hline Characteristic & $\begin{array}{l}\leq 0 \% \text { viable tumor } \\
(n=12)\end{array}$ & $\begin{array}{l}>10 \% \text { viable tumor } \\
(n=19)\end{array}$ & $p$ value \\
\hline Neoadjuvant regimens & & 16 & 0.999 \\
$\quad$ Chemo-immunotherapy & 11 & 3 & \\
$\quad$ Immunotherapy alone & 1 & & 0.552 \\
Prescribed checkpoint inhibitor & 5 & 10 & \\
Pembrolizumab & 7 & 9 & 0.782 \\
Nivolumab & $3(2-3)$ & $2(2-4)$ & 0.314 \\
Median doses (range) & $32(4-93)$ & $36(23-86)$ & 0.352 \\
Median duration from final treatment to & & & \\
$\quad$ surgery (range), days & 10 & 14 & \\
Radiographic response assessment & 2 & 3 & \\
PR & 0 & 2 & \\
SD & & & \\
PD & & & \\
\hline
\end{tabular}

$P R$ partial response; $S D$ stable disease; $P D$ progressive disease
(2, 6.5\%). Minimally invasive approaches, including video-assisted thoracic surgery (VATS) in 8 patients, and robot-assisted thoracic surgery (RATS) in 1 patient, were attempted in 9 resections (7 lobectomies, 1 sleeve lobectomy, 1 bilobectomy). In total, only 1 patient (3.2\%) with clinical stage IIB tumor, who was initially attempted to underwent VATS procedure, was converted to open thoracotomy due to dense adhesions of hilar lymph node causing difficulty dissecting the blood vessel. Therefore, the conversion rate from minimally invasive procedures to thoracotomy in the present population was $3.2 \%$. Median operative time was $158 \mathrm{~min}$ (range, 77-279 min). Median estimated blood loss was $200 \mathrm{~mL}$ (range, 50-1600 mL). Two patients (\%) required blood transfusion. Median hospital length of stay after surgery was 7 days (range, 2-29 days).

Final pathological examination demonstrated 12 of 31 patients (38.7\%) had a major pathological response (MPR) (defined as $\leq 10 \%$ residual viable tumor), 15 (48.4\%) pathological downstaging. Three patients $(9.7 \%)$ had no residual viable tumor, which means pathological complete remission, while viable tumor cells in 28 patients (90.3\%) (Fig. 2). A positive surgical margin (R1 or R2) was identified in 7 (22.6\%) cases, including 4 cases of $\mathrm{R} 1$ resection and 3 cases of $\mathrm{R} 2$ resection (Table 3 ). All 4 patients were classified as R1 resections because of positive highest mediastinal lymph nodes. Even though intraoperative pathology was conducted as clinical routine activity, positive surgical margin status was inevitable in these 3 patients identified as R2 resection, due to their limited lung function intolerant of bilobectomy or pneumonectomy. One or more postoperative complications occurred in 18 of all 31 patients (overall morbidity, 58.1\%) (Table 3). The most common complication was prolonged air leak, occurring in 14 patients (45.2\%), followed by arrhythmia ( $n=3,9.7 \%)$, pneumonia $(n=1,3.2 \%)$, chylothorax $(n=1,3.2 \%)$ and wound infection $(n=1,3.2 \%)$. No early deaths (within 90 days) were reported among the whole cohort. There was no significant difference in complication rates between the two groups, patients had $\leq 10 \%$ viable tumor remaining on final pathological assessment, and those who had $>10 \%$ viable tumor $(p=0.981)$.

Following completion of surgical therapy, 11 patients (35.5\%) received adjuvant chemo-immunotherapy, 5 patients (16.1\%) chemotherapy, 4 patients $(12.9 \%)$ chemoradiotherapy, 1 patient (3.2\%) postoperative radiotherapy, 1 patient (3.2\%) immunotherapy alone, 1 patient $(3.2 \%)$ targeted therapy, while the remaining 8 patients $(25.8 \%)$ underwent active surveillance. Remarkably, for these 7 patients with positive surgical margin (R1 or R2), postoperative radiotherapy or chemoradiotherapy was conducted following NCCN guidelines. Totally, 19 of 31 patients (61.3\%) in the current cohort discontinued checkpoint inhibitor treatment postoperatively. Notably, one female 57-year-old patient diagnosed as stage IIIA adenocarcinoma, whose initial gene assay showed wild-type EGFR and KRAS, negative for ALK and ROS1 gene rearrangement, was prescribed chemo-immunotherapy (carboplatinpemetrexed and nivolumab). After 3 doses neoadjuvant treatment, right upper lobe lobectomy was conducted. It is interesting that postoperative gene assay indicated EGFR exon 19 deletion. Therefore, adjuvant targeted therapy was suggested to this individual.

Additionally, 26 patients underwent next-generation sequencing (NGS) before surgery in total (Figs. 1, 2). Among them, 2 patients $(7.7 \%)$ were detected STK11 mutations. None of these 2 individuals had an MPR by final pathological examination, which also confirmed that 
Table 3 Surgical and postoperative characteristics of the overall cohort

\begin{tabular}{|c|c|c|c|}
\hline Characteristic & $\begin{array}{l}\leq 10 \% \text { viable tumor } \\
(n=12)\end{array}$ & $\begin{array}{l}>10 \% \text { viable tumor } \\
(n=19)\end{array}$ & $p$ value \\
\hline Tumor location & & & 0.250 \\
\hline LUL & 2 & 3 & \\
\hline LLL & 1 & 2 & \\
\hline RUL & 5 & 12 & \\
\hline RML & 0 & 1 & \\
\hline RLL & 4 & 1 & \\
\hline Extent of resection & & & 0.181 \\
\hline Lobectomy & 4 & 14 & \\
\hline Bilobectomy & 3 & 1 & \\
\hline Sleeve lobectomy & 5 & 2 & \\
\hline Pneumonectomy & 0 & 2 & \\
\hline Approach & & & 0.930 \\
\hline Open thoracotomy & 9 & 13 & \\
\hline VATS & 2 & 6 & \\
\hline RATS & 1 & 0 & \\
\hline Median operative time (range), min & $159(77-223)$ & $157(82-279)$ & 0.994 \\
\hline Median estimated blood loss (range), $\mathrm{ml}$ & $200(50-200)$ & $200(50-1600)$ & 0.265 \\
\hline $\begin{array}{l}\text { Median hospital length of stay after surgery } \\
\text { (range), days }\end{array}$ & $6(2-25)$ & $8(4-29)$ & 0.906 \\
\hline Pathological downstaging & & & 0.106 \\
\hline No & 4 & 12 & \\
\hline Yes & 8 & 7 & \\
\hline Surgical margin & & & 0.029 \\
\hline R0 & 12 & 12 & \\
\hline $\mathrm{R} 1$ & 0 & 4 & \\
\hline $\mathrm{R} 2$ & 0 & 3 & \\
\hline Thirty-day mortality & 0 & 0 & NA \\
\hline Ninety-day mortality & 0 & 0 & NA \\
\hline Postoperative complications & & & 0.749 \\
\hline Prolonged air leak & 6 & 8 & \\
\hline Arrhythmia & 1 & 2 & \\
\hline Pneumonia & 0 & 1 & \\
\hline Chylothorax & 1 & 0 & \\
\hline Wound infection & 0 & 1 & \\
\hline Adjuvant treatment & & & 0.068 \\
\hline Chemo-immunotherapy & 4 & 7 & \\
\hline Chemotherapy & 3 & 2 & \\
\hline Chemoradiotherapy & 0 & 4 & \\
\hline Radiotherapy & 0 & 1 & \\
\hline Immunotherapy alone & 1 & 0 & \\
\hline Targeted therapy & 0 & 1 & \\
\hline Active surveillance & 4 & 4 & \\
\hline
\end{tabular}

$L U L$ left upper lobe; $L L L$ left lower lobe; $R U L$ right upper lobe; $R M L$ right middle lobe; $R L L$ right lower lobe; VATS video-assisted thoracic surgery; RATS robot-assisted thoracic surgery; $N A$ not applicable
STK11 mutations might be an unfavorable factor to neoadjuvant immunotherapy [14] (Figs. 1, 2).

\section{Discussion}

In the current study, it is safe and feasible of the application of neoadjuvant immunotherapy or chemo-immunotherapy before surgery for patients with resectable NSCLC. 


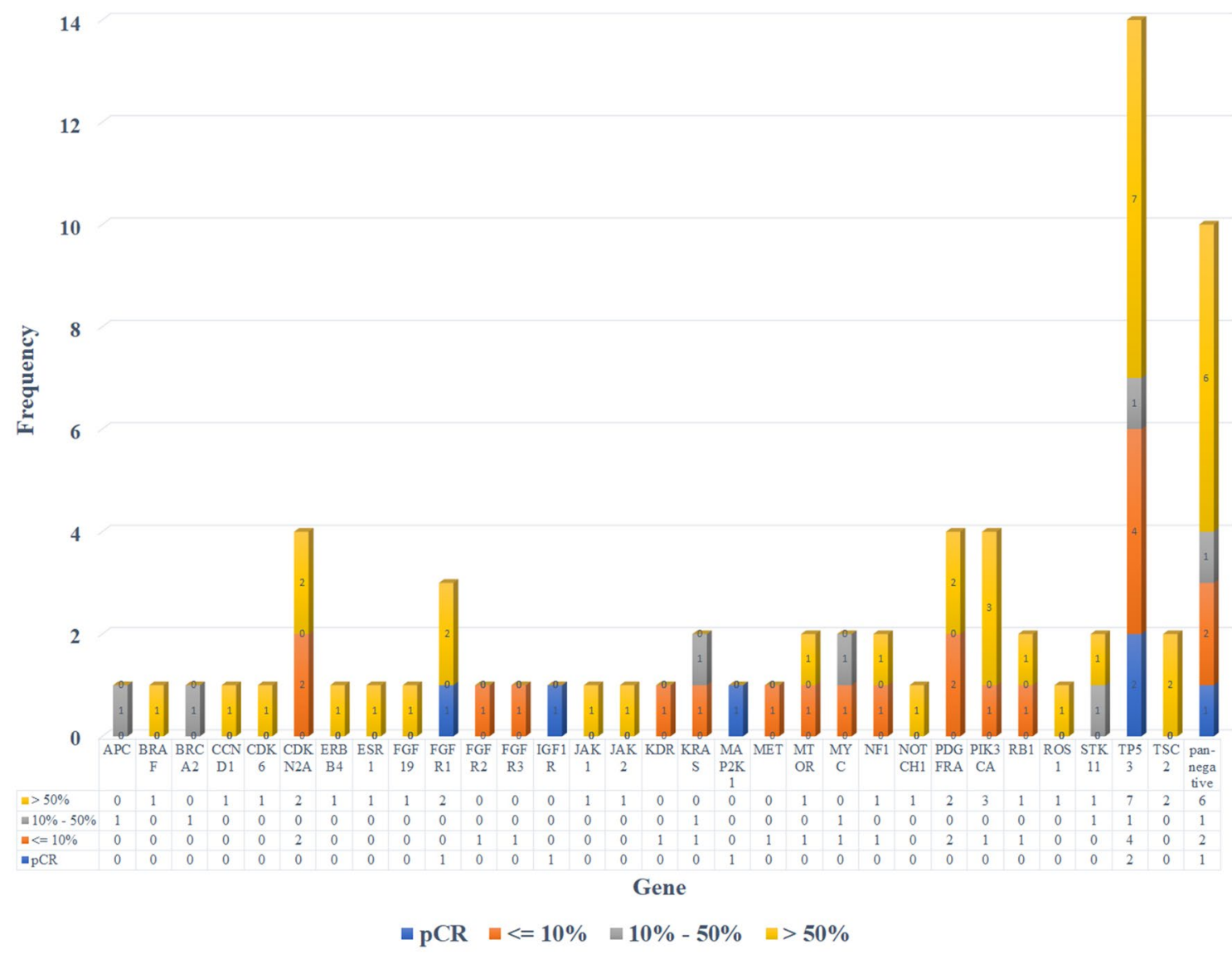

Fig. 2 Detailed information about pathological response of 26 patients with NGS result. NGS next-generation sequencing

As is well known, with the rapid development of modern tumor immunology, immunotherapy has become an increasingly important weapon and achieving great success in tumor therapy [23]. The role of immunotherapy has restructured the treatment approach to numerous malignancies. Immune checkpoint inhibitors have revolutionized the therapeutic landscape in oncology and have shown significant clinical benefit in several cancer types [24]. The clinical application of immune checkpoint inhibitors has dramatically changed the treatment landscape for patients with advanced cancers, including advanced NSCLC [25].

Prior to immunotherapy, treatment for advanced NSCLC had not changed significantly since the broad uptake of chemotherapy over best supportive care in the mid-1990s [26]. Neoadjuvant chemotherapy has already been widely accepted as a comprehensive therapeutic strategy, especially for local advanced lung cancer [27]. Immunotherapy with immune checkpoint inhibitors has brought real progress in the treatment of solid tumors including lung cancer, which have revolutionized the management of patients with NSCLC [28]. At present, numerous ongoing investigations of neoadjuvant immunotherapy or chemo-immunotherapy demonstrate the promising future of immunotherapy in the complex management paradigm of advanced NSCLC, which represents one of the next frontiers in cancer immunotherapy $[10,29,30]$.

With concerns of potential pulmonary and extrapulmonary toxicity after immunotherapy administration, such as pneumonitis, adrenal insufficiency and hyperthyroidism [31], for patients who have received neoadjuvant immunotherapy or chemo-immunotherapy, the safety of following pulmonary resection is the primary concern for thoracic surgeons [19]. According to previous studies, secondary analyses of former small pilot prospective studies, the approach of lung resection after neoadjuvant therapy is safe and reliable $[10,11,32]$. Our initial series demonstrated low rates of mortality and major intraoperative and postoperative complications, similar to those published in previous neoadjuvant immunotherapy or chemotherapy settings [19, 27]. In our current cohort, the rates of chest tube air leak (45.2\%), arrhythmia $(n=3,9.7 \%)$, pneumonia $(3.2 \%)$, reintubation $(0 \%)$ and respiratory failure $(0 \%)$ were acceptable and comparable to previous studies $[19,27]$. Compared with patients without neoadjuvant treatment, the complication profile was similar in the current population. The complications occurred at similar levels in the present neoadjuvant group, 
comparable with previous reports of without neoadjuvant cohort as $42.5-68.3 \%$ [33]. In addition, a similar trend is observed for the distribution of postoperative complications between the two systems [33]. In summary, patients who underwent surgery after neoadjuvant immunotherapy or chemo-immunotherapy did not have out-of-range intraoperative and postoperative complications rate, which indicates that patients can be safely operated with satisfied surgical outcomes after prescribed neoadjuvant immunotherapy or chemotherapy agents.

Since it is hard to quantitatively evaluate the difficulty of the surgery, there are some significant manifestations, such as operating time, amount of blood loss, conversion rate and perioperative complications [21]. In the present study, all similar manifestations as previously reported in the literature $[11,19,27,32]$, including comparable operation time, minimal intraoperative blood loss, low level of conversion rate and low rates of mortality and complications, indicate that neoadjuvant immunotherapy or chemo-immunotherapy does not increase the complexity and difficulty of the operation.

As one of the most significant predictors of prolonged survival, radical (R0) resection rate is critical to underline the feasibility and utility of neoadjuvant therapeutical strategy $[27,34]$. Expect for the $77.4 \%$ R0 resection rate in the present cohort, it is worth noting that 12 patients (38.7\%) have no evidence of residual tumor cell at microscopic examination. The present pilot data confirmed that STK11 mutations might be an unfavorable factor to neoadjuvant immunotherapy [14]. Consequently, the identification and characterization of novel markers that can predict residual disease after neoadjuvant therapy is critical to investigate new management approaches to select appropriate patient and improve clinical outcome.

As a real-world study focusing on patients with clinical practice, instead of clinical trial, the current report suggests the safety of surgical resection after neoadjuvant immunotherapy or chemo-immunotherapy in resectable NSCLC patients. Considering that neoadjuvant immunotherapy continues to be studied both individually and in combination with chemotherapy, these results would have a pertinence for thoracic oncologists and surgeons.

What remains unclear is the optimum neoadjuvant doses and interval from final administration of neoadjuvant agents to operation. In the situation of lacking evidence-based algorithms, current study could provide implications for clinical practice. Based on our data, neoadjuvant immunotherapy or chemo-immunotherapy was prescribed for 2-4 courses, followed by radiographic assessment of residual cancer and treatment response. After the last prescription, patients were often observed for a drug-free interval period of approximately 30 days to ensure they are fully prepared for the subsequent surgery. In the current cohort, two patients experienced a relevant long drug-free interval of 86 and 93 days, because of the Chinese Lunar New Year holiday and COVID-19 pandemic since January 2020 [35]. However, after control of COVID-19 outbreak in China [36], both of them were admitted and underwent lobectomy and sleeve lobectomy.

The study involved several limitations. The present retrospective observational study has inherent limitations. Although there is an increasing number of patients who underwent surgery following immunotherapy, sufficiently large sample size report is lacking, and enough experiences still expected. Future research including prospective data and follow-up assessments is in progress.

Conclusively, pulmonary resection after neoadjuvant immunotherapy or chemo-immunotherapy for resectable NSCLC appears to be safe with low operative mortality and morbidity rate in the current population. Because of possible post-neoadjuvant treatment adhesions or fibrosis, concerns that surgical treatment for these patients might be challenging have raised. However, based on the current data, difficulty of operation appears to be similar to those treated with other neoadjuvant agents. Future studies with larger sample numbers will address new perspectives in the setting of neoadjuvant immunotherapy or chemo-immunotherapy.

Acknowledgements We wish to thank Prof Pamela Derish, Scientific Publications Manager of UCSF Department of Surgery (University of California, San Francisco), for language editing. The Institutional Review Board of Shanghai Chest Hospital approved the retrospective analysis of anonymous patient data.

Author contributions LJ, JH, SSJ and QQL conceived the presented idea and designed the work. LJ, WWR and YFS conducted the data collection. LJ, CWL and YT performed the data analysis and interpretation. LJ and JWN drafted the article. LJ, XKC, YHY, ZPD and QQL conducted the operative procedures. ZML prescribed the neoadjuvant immunotherapy or chemo-immunotherapy. QQL encouraged to investigate and supervised the findings of this work. All authors discussed the results and contributed to the final manuscript.

Funding This work was supported by the National Natural Science Foundation of China (No. 81702251, 81972176 and 81800073), the Science Foundation of Shanghai (No.18ZR1435100) and Shanghai Hospital Development Center (SHDC12016113).

Data availability material The data that support the findings of this study are available on request from the corresponding author. The data are not publicly available due to privacy or ethical restrictions.

\section{Compliance with ethical standards}

Conflict of interest The authors declare that they have no competing interests.

Informed consent Not required. 


\section{References}

1. Siegel RL, Miller KD, Jemal A (2020) Cancer statistics, 2020. CA Cancer J Clin. 70:7-30. https://doi.org/10.3322/caac.21590

2. Bagcchi S (2017) Lung cancer survival only increases by a small amount despite recent treatment advances. Lancet Respir Med. 5:169. https://doi.org/10.1016/S2213-2600(17)30041-3

3. Herbst RS, Morgensztern D, Boshoff C (2018) The biology and management of non-small cell lung cancer. Nature. 553:446-54. https://doi.org/10.1038/nature25183

4. Hirsch FR, Scagliotti GV, Mulshine JL, Kwon R, Curran WJ Jr, Wu YL, Paz-Ares L (2017) Lung cancer: current therapies and new targeted treatments. Lancet. 389:299-311. https://doi. org/10.1016/S0140-6736(16)30958-8

5. Garon EB, Hellmann MD, Rizvi NA et al (2019) Five-year overall survival for patients with advanced nonsmall-cell lung cancer treated with pembrolizumab: results from the phase I KEYNOTE-001 Study. J Clin Oncol. 37:2518-27. https://doi. org/10.1200/JCO.19.00934

6. Gandhi L, Rodriguez-Abreu D, Gadgeel S et al (2018) Pembrolizumab plus chemotherapy in metastatic non-small-cell lung cancer. N Engl J Med. 378:2078-92. https://doi.org/10.1056/ NEJMoa1801005

7. DeBerardinis RJ (2020) Tumor microenvironment, metabolism, and immunotherapy. N Engl J Med. 382:869-71. https://doi. org/10.1056/NEJMcibr1914890

8. Lin JJ, Shaw AT (2017) Raising the bar on first-line immunotherapy in lung cancer. Lancet Oncol. 18:2-3. https://doi. org/10.1016/S1470-2045(16)30594-0

9. Ettinger DS, Wood DE, Aggarwal C et al (2019) NCCN guidelines Insights: non-small cell lung cancer, version 1.2020. J Natl Compr Canc Netw. 17:1464-72. https://doi.org/10.6004/jnccn 2019.0059

10. Forde PM, Chaft JE, Smith KN et al (2018) Neoadjuvant PD-1 blockade in resectable lung cancer. N Engl J Med. 378:1976-86. https://doi.org/10.1056/NEJMoa1716078

11. Bott MJ, Yang SC, Park BJ et al (2019) Initial results of pulmonary resection after neoadjuvant nivolumab in patients with resectable non-small cell lung cancer. J Thorac Cardiovasc Surg. 158:269-76. https://doi.org/10.1016/j.jtcvs.2018.11.124

12. Provencio M, Nadal E, Insa A et al (2018) OA01.05 phase II study of neo-adjuvant chemo/immunotherapy for resectable stages IIIA non-small cell lung cancer- Nadim studySLCG. J Thoracic Oncol. 13:320. https://doi.org/10.1016/j. jtho.2018.08.236

13. Altorki N, Borczuk A, Saxena A et al (2019) P2.04. J Thoracic Oncol. 14:746. https://doi.org/10.1016/j.jtho.2019.08.1597

14. Shu CA, Gainor JF, Awad MM et al (2020) Neoadjuvant atezolizumab and chemotherapy in patients with resectable non-smallcell lung cancer: an open-label, multicentre, single-arm, phase 2 trial. Lancet Oncol. 21:786-95. https://doi.org/10.1016/S1470 $-2045(20) 30140-6$

15. Cascone T, William WN, Weissferdt A et al (2019) Neoadjuvant nivolumab $(\mathrm{N})$ or nivolumab plus ipilimumab (NI) for resectable non-small cell lung cancer (NSCLC): clinical and correlative results from the NEOSTAR study. J Clin Oncol 37:8504. https://doi.org/10.1200/JCO.2019.37.15_suppl.8504

16. Kwiatkowski DJ, Rusch VW, Chaft JE et al (2019) Neoadjuvant atezolizumab in resectable non-small cell lung cancer (NSCLC): Interim analysis and biomarker data from a multicenter study (LCMC3). J Clin Oncol 37:8503. https://doi. org/10.1200/JCO.2019.37.15_suppl.8503

17. Provencio M, Nadal E, Insa A et al (2019) Neoadjuvant chemoimmunotherapy for the treatment of stage IIIA resectable non-small-cell lung cancer (NSCLC): A phase II multicenter exploratory study - final data of patients who underwent surgical assessment. J Clin Oncol 37:8509. https://doi.org/10.1200/ JCO.2019.37.15_suppl.8509

18. Broderick SR (2020) Adjuvant and neoadjuvant immunotherapy in non-small cell lung cancer. Thorac Surg Clin. 30:215-20. https://doi.org/10.1016/j.thorsurg.2020.01.001

19. Broderick SR, Bott MJ (2019) Neoadjuvant immunotherapy in patients with resectable non-small cell lung cancer. J Thorac Cardiovasc Surg. 158:1471-4. https://doi.org/10.1016/j.jtcvs .2019.06.114

20. Goldstraw P, Chansky K, Crowley J et al (2016) The IASLC Lung cancer staging project: proposals for revision of the TNM stage groupings in the forthcoming (eighth) edition of the TNM classification for lung cancer. J Thorac Oncol. 11:39-51. https ://doi.org/10.1016/j.jtho.2015.09.009

21. Fernandez FG, Falcoz PE, Kozower BD, Salati M, Wright CD, Brunelli A (2015) The society of thoracic surgeons and the European Society of Thoracic Surgeons general thoracic surgery databases: joint standardization of variable definitions and terminology. Ann Thorac Surg. 99:368-76. https://doi. org/10.1016/j.athoracsur.2014.05.104

22. Hellmann MD, Chaft JE, William WN Jr et al (2014) Pathological response after neoadjuvant chemotherapy in resectable nonsmall-cell lung cancers: proposal for the use of major pathological response as a surrogate endpoint. Lancet Oncol. 15:e42-50. https://doi.org/10.1016/S1470-2045(13)70334-6

23. Popovic A, Jaffee EM, Zaidi N (2018) Emerging strategies for combination checkpoint modulators in cancer immunotherapy. J Clin Invest. 128:3209-18. https://doi.org/10.1172/JCI120775

24. Havel JJ, Chowell D, Chan TA (2019) The evolving landscape of biomarkers for checkpoint inhibitor immunotherapy. Nat Rev Cancer. 19:133-50. https://doi.org/10.1038/s41568-019-0116-x

25. Ribas A, Wolchok JD (2018) Cancer immunotherapy using checkpoint blockade. Science. 359:1350-5. https://doi. org/10.1126/science.aar4060

26. Non-small Cell Lung Cancer Collaborative Group (1995) Chemotherapy in non-small cell lung cancer: a meta-analysis using updated data on individual patients from 52 randomised clinical trials. Non-small Cell Lung Cancer Collaborative Group BMJ 311:899-909

27. Gilligan D, Nicolson M, Smith I et al (2007) Preoperative chemotherapy in patients with resectable non-small cell lung cancer: results of the MRC LU22/NVALT 2/EORTC 08012 multicentre randomised trial and update of systematic review. Lancet. 369:1929-37. https://doi.org/10.1016/S0140-6736(07)60714-4

28. Hellmann MD, Nathanson T, Rizvi H et al (2018) Genomic features of response to combination immunotherapy in patients with advanced non-small-cell lung cancer. Cancer Cell. 33(84352):e4. https://doi.org/10.1016/j.ccell.2018.03.018

29. Eichhorn F, Klotz LV, Bischoff H, Thomas M, Lasitschka F, Winter H, Hoffmann H, Eichhorn ME (2019) Neoadjuvant anti-programmed death-1 immunotherapy by pembrolizumab in resectable nodal positive stage II/IIIa non-small-cell lung cancer (NSCLC): the NEOMUN trial. BMC Cancer. 19:413. https://doi.org/10.1186/s12885-019-5624-2

30. Gao S, Li N, Gao S et al (2020) Neoadjuvant PD-1 inhibitor (Sintilimab) in NSCLC. J Thorac Oncol. 15:816-26. https:// doi.org/10.1016/j.jtho.2020.01.017

31. Kennedy LB, Salama AKS (2020) A review of cancer immunotherapy toxicity. CA Cancer J Clin. 70:86-104. https://doi. org/10.3322/caac. 21596

32. Yang CJ, McSherry F, Mayne NR et al (2018) Surgical outcomes after neoadjuvant chemotherapy and ipilimumab for nonsmall cell lung cancer. Ann Thorac Surg. 105:924-9. https://doi. org/10.1016/j.athoracsur.2017.09.030 
33. Thorsteinsson H, Alexandersson A, Oskarsdottir GN, Skuladottir R, Isaksson HJ, Jonsson S, Gudbjartsson T (2012) Resection rate and outcome of pulmonary resections for non-small-cell lung cancer: a nationwide study from Iceland. J Thorac Oncol. 7:1164-9. https://doi.org/10.1097/JTO.0b013e318252d022

34. Dy GK, Bogner PN, Tan W et al (2014) Phase II study of perioperative chemotherapy with cisplatin and pemetrexed in nonsmall-cell lung cancer. J Thorac Oncol. 9:222-30. https://doi. org/10.1097/JTO.0000000000000062

35. Pan A, Liu L, Wang C et al (2020) Association of public health interventions with the epidemiology of the COVID-19 outbreak in Wuhan. JAMA, China. https://doi.org/10.1001/ jama.2020.6130
36. Chen S, Yang J, Yang W, Wang C, Barnighausen T (2020) COVID-19 control in China during mass population movements at New Year. Lancet. 395:764-6. https://doi.org/10.1016/S0140 $-6736(20) 30421-9$

Publisher's Note Springer Nature remains neutral with regard to jurisdictional claims in published maps and institutional affiliations. 